(C) 2016. This manuscript version is made available under the CC-BY-NC-ND 4.0 license http://creativecommons.org/licenses/by-nc-nd/4.0/

\title{
Power set extensions of dichotomous preferences*
}

\author{
Ritxar Arlegi \\ Department of Economics, Public University of Navarre, Spain \\ and
}

INARBE, Institute for Advanced Research in Bussiness and Economics, Spain

e-mail: rarlegi@unavarra.es

\section{Dinko Dimitrov}

Chair of Economic Theory, Saarland University, Germany

e-mail: dinko.dimitrov@mx.uni-saarland.de

\begin{abstract}
In this paper we assume that a set $X$ is partitioned into two indifference classes and study several ways in which this dichotomous information can be extended, so as to obtain an ordering of the power set of $X$. The axioms that we introduce enable such a partition of $X$ to be interpreted as being made out of "good" and "bad" objects. We define a family of rules that naturally take into account the number of
\end{abstract}

*We thank two anonymous referees for their insightful suggestions. R. Arlegi gratefully acknowledges financial support from the Spanish Ministry of Education, projects ECO2012-34202 and ECO2015-65031-R, as well as helpful comments by Nuria Osés and Jon Benito. 
good objects and the number of bad objects, and provide axiomatic characterizations of different rules in the family that fit into a variety of decisional situations.

JEL Classification Number: D81.

Keywords: dichotomous preferences, ranking sets, infection, opportunity sets, freedom of choice.

\section{Introduction}

In this paper we apply an axiomatic approach to the study of the question of how a decision maker ranks sets of objects in contexts where the primitive information held about them is dichotomous. By this we mean that the decision maker partitions the set of all objects into two indifference classes, and considers only this information in order to evaluate and rank sets. As shown below, the axioms that we introduce enable the partition to be reinterpreted as consisting of "good" and "bad" objects.

Categorization, and in particular dichotomous categorization, is a wellknown phenomenon in cognitive and social psychology, especially since Allport (1954). In general, it is understood that categorization helps to handle and process information efficiently in numerous decision processes. As we illustrate below, there is a considerable number of settings where both dichotomous categorization and the ranking of sets on the basis of such dichotomous partitions are compelling.

For instance, as extensively surveyed by Brams and Fishburn (2002), dichotomous domains have been examined in the context of voting, and in particular in the case of approval voting, where voting strategies are reported precisely in the form of sets of (approved) candidates. In such a context dichotomous domains turn out to have important theoretical implications regarding, for example, sincere and strategy-proof voting. 
Similarly, in problems of the voting by committees type initially posed by Barberà et al. (1991), the basic item of information concerns the voter's preferences over subsets of candidates, and as in approval voting it makes full sense to think about situations where voters categorize candidates dichotomously, for example in terms of acceptable versus non-acceptable alternatives.

Bogomolnaia and Moulin (2004) also study bilateral matchings where each side partitions the set of potential partners into "acceptable" and "nonacceptable" (for example, depending on whether they have certain skills or qualifications). It is, for instance, easy to imagine many-to-one matching problems, such as designing working teams, where preferences of the "oneside" of the market over groups of candidates have to be derived from their basic dichotomous preferences over single candidates. Furthermore, Barberà et al. (2001), Dimitrov et al. (2007), Kasher and Rubinstein (1997), and Samet and Schmeidler (2003) study society formation problems, in which the distinction is between candidates who qualify for membership on the basis of the opinion of a founder or member of the society - for example based on religious or political principles - and those who do not qualify. In other cases, the members of a society have to decide who is entitled to perform a certain activity within the group, such as driving a car or teaching at a university.

Groups may also be evaluated from outside. For example, in a social dilemma situation such as a public good game, an individual could evaluate the convenience of joining alternative coalitions on the basis of his/her knowledge regarding the existence of two possible types of agent in the coalitions: cooperators and free-riders. ${ }^{1}$

\footnotetext{
${ }^{1}$ Our dichotomous approach obviously makes more sense (though not necessarily) when agents have only two possible strategies, i.e. either to contribute a given amount of money, level of effort, etc. or not to contribute at all, as in the standard prisoner's dilemma or in all-or-none public good games.
} 
The results of our approach can also be seen as a contribution to the problem of ranking sets of objects in a context of choice under complete uncertainty, or ignorance. In these problems, the probabilities of the outcomes generated by each action are totally unknown or ignored. Therefore, each individual decision is simply described by the set of outcomes that it generates (see, among others, Arlegi (2003, 2007), Barberà et al. (1984), Barberà and Pattanaik (1984), Bossert et al. (2000) and Nitzan and Pattanaik (1984)). If it is assumed that the future uncertain outcomes are dichotomous (as in Bogomolnaia et al. (2005)) and that assumption is considered within an environment of complete uncertainty, the problem to be analyzed collapses again into the formal structure that we propose.

When evaluating the freedom of choice that an opportunity set provides it is sometimes the case that there is a partition between opportunities that increase the freedom of the agent and those that do not (see Romero-Medina (2001)).

As can be seen, it is not difficult to imagine very diverse types of problem that consist of evaluating sets of items on the basis of a dichotomous partition of the set of single items. Further cases could be those of an individual or group interested in evaluating sets of norms according to whether or not they comply with a certain moral doctrine; a worker that has to decide about alternative day/night shifts proposed by his/her employer; a company that evaluates alternative suppliers of a certain component according to their ratios of $\mathrm{OK} / \mathrm{NOK}$ items delivered, etc.

As explained above, whatever the context considered, the basic specific question that we ask is the following: under a setting where the preferences over individual objects are dichotomous, how can this rudimentary information about the preferences on a set of objects be meaningfully extended to obtain an ordering of the power set of those objects? Section 2 below presents the basic notation and definitions. We then answer this question in Section 
3 by introducing three core axioms which are suitable under the different contexts described above. These axioms naturally allow us to concentrate on dichotomous preferences where the two types of object can be interpreted not just as belonging to two indifferent classes but as good and bad, respectively. It turns out that the requirements that we impose shape a specific family of rules for ranking sets in our dichotomous environment. In Section 4 we then present axiomatic characterizations of different rules that belong to the family defined, with each rule accounting for the number of good objects and the number of bad objects in the corresponding sets under comparison, but differing in the way in which the two numbers are combined.

\section{Notation and definitions}

We start by defining a dichotomous domain following Brams and Fishburn (1978). Let $X$ be a universal finite set of objects and $R$ a binary relation defined on $X$. We denote the symmetric and asymmetric parts (indifference and strict preference) of $R$ by $I$ and $P$, respectively. $R$ is called dichotomous if there exists a partition $\left(A_{1}, A_{2}\right)$ of $X, A_{i} \neq \emptyset, i=1,2$, such that $a I b$ holds for $a, b \in A_{i}, i=1,2$, and $a P b$ holds for $a \in A_{1}$ and $b \in A_{2}{ }^{2}$

Let $\mathcal{X}$ be the set of all subsets of $X$ including the empty set. In our model, the elements of $\mathcal{X}$ are the (alternative) sets of objects that an agent may be confronted with. The question now arises of how this agent compares sets using only the information provided by $R$. Consequently, the problem to be analyzed is how to establish a binary relation $\succcurlyeq$ defined on $\mathcal{X}$. At this point we assume that $\succcurlyeq$ is reflexive and transitive, but not necessarily complete. When assuming only reflexivity and transitivity we will say that $\succcurlyeq$ is a ranking over the sets, while when assuming that it is also complete

\footnotetext{
${ }^{2}$ As the reader can easily check, any dichotomous binary relation is reflexive, complete, and transitive.
} 
we will say that it is a complete ranking. ${ }^{3}$ For all $C, D \in \mathcal{X}, C \succcurlyeq D$ is interpreted as " $C$ is at least as good as $D$ ". The asymmetric and symmetric factors of $\succcurlyeq$ are denoted by $\succ$ ("is strictly better than") and $\sim$ ("is as good as"), respectively. We say that $\succcurlyeq$ is an extension of $R$ if for $x, y \in X, x R y$ implies $\{x\} \succcurlyeq\{y\} .4$

Now let $\{X\} \subset \mathcal{X}$ be the set of all singletons in $\mathcal{X}$. We say that $\succcurlyeq$ is based on a dichotomous partition between good and bad singletons, and call it a $g b$-ranking, if there exists a partition $\left(A_{1}^{*}, A_{2}^{*}\right)$ of $\{X\}, A_{i}^{*} \neq \emptyset, i=1,2$, such that $\{a\} \sim\{b\}$ holds for $\{a\},\{b\} \in A_{i}^{*}, i=1,2$, and $\{a\} \succ \emptyset \succ\{b\}$ holds for $\{a\} \in A_{1}^{*}$ and $\{b\} \in A_{2}^{*}$.

The idea behind a $g b$-ranking is that of a transitive and reflexive binary relation over sets where there are two indifference classes of singletons, $A_{1}^{*}$ and $A_{2}^{*}$. The singletons in $A_{1}^{*}$ are strictly better than the empty set and those in $A_{2}^{*}$ are strictly worse. Thus, the definition of a $g b$-ranking enables the corresponding elements from $A_{1}^{*}$ to be interpreted as being "good" and those from $A_{2}^{*}$ as being "bad". Clearly, by the definition of extension, if $R$ is dichotomous then it directly induces a dichotomous preference over the set of singletons. But it is worth mentioning that not every extension $\succcurlyeq$ of a dichotomous preference $R$ is a $g b$-ranking. The reason here is that if it is assumed solely that $\succcurlyeq$ is an extension of $R$ then it is not known where the empty set should be placed. Thus, one needs additional requirements such as those described in the next section so as to induce a $g b$-ranking over sets by extending a dichotomous preference $R$ over objects. ${ }^{5}$

\footnotetext{
${ }^{3}$ All the characterization results of the following sections hold for incomplete binary relations except Theorem 7 .

${ }^{4}$ As Barberà et al. (2004) show in their survey on ranking sets models, this property is often presented as a minimal axiomatic requirement for extending a preference over a set of elements to a preference over its possible subsets.

${ }^{5}$ Notice that if $\succcurlyeq$ is an extension of a dichotomous preference $R$ or a $g b$-ranking, then it is complete over the set of all singletons in $\mathcal{X}$.
} 


\section{A family of rules}

We start our analysis by introducing three axioms that will constitute the axiomatic core of the results that we propose throughout the sections below. Local Positive Response (LPR): There exists $A \in \mathcal{X}$ such that $A \cup\{x\} \succ A$ for some $x \in X \backslash A$.

Local Negative Response (LNR): There exists $A \in \mathcal{X}$ such that $A \cup\{x\} \prec A$ for some $x \in X \backslash A$.

Independence (IND): For all $A, B \in \mathcal{X}$, and all $x \in X \backslash(A \cup B), A \succcurlyeq B \Leftrightarrow$ $A \cup\{x\} \succcurlyeq B \cup\{x\}$.

Axiom LPR states that a set $A$ and an external element $x$ can always be found such that $A$ is improved by the addition of $x$. The adjective "local" comes from the fact that LPR only requires that there be at least one set such that, for at least one external element that is added to it, the set becomes strictly better. This condition is weaker than assuming the existence of what Barberà et al. (1991), in the context of voting by committees, define as a "good" element, i.e. an element that, as a singleton, is strictly better than the empty set. On the other hand, if the degenerate case where the basic preference defined on $X$ is the universal indifference is disregarded, LPR is also weaker than condition $P$ in Brams and Fishburn (1978) which, in a context of approval voting, states that, for $x, y \in X,\{x\} \succ\{x, y\} \succ$ $\{y\}$ if and only if $x P y$. The interpretation and scope of axiom LNR are straightforward as a dual of LPR. Notice that LRP and LNR can also be seen as incorporating two natural requirements for $\succcurlyeq$ being minimally rich.

On the other hand, IND refers to the effect of adding the same element to (or dropping it from) a pair of sets $A$ and $B$. In particular, the axiom says that the original ranking between the two sets is preserved under such a modification. This axiom imposes an idea of separability that is widely 
used in ranking sets models under different settings. However it should be warned that such a separability idea in fact forces the elements in the set not to have any specific correlation, which in some contexts can be considered as not very realistic. In some studies this kind of separability idea is expressed exactly as the axiom that we propose here is (for example, Pattanaik and Xu (1990) and Bossert et al. (1994) among many others). Other studies use related properties. For example, IND is a stronger version of the following separability condition proposed by Barberà et al. (1991): for $A \in \mathcal{X} \backslash \emptyset$ and $x \in X \backslash A,\{x\} \succ \emptyset \Leftrightarrow A \cup\{x\} \succ A$.

Theorem 1 Let $R$ be a dichotomous preference on $X$ and $\succcurlyeq$ be an extension of it. If $\succcurlyeq$ satisfies IND, LPR and $L N R$, then it is a gb-ranking.

As noted above, starting from a dichotomous preference $R$ and merely extending it does not necessarily lead to a $g b$-ranking. What can be obtained is at most a ranking with two indifference classes among the singletons, but with no connotation of absolute "goodness" or "badness" attachable to them. Theorem 1 establishes IND, LPR and LNR as sufficient conditions to ensure that the extension from a dichotomous preference $R$ to a ranking over sets results in a $g b$-ranking. The proof, like those of all other results except that of Theorem 2, is presented in the Appendix. ${ }^{6}$

Theorem 1 only establishes a logical implication in one direction. As for the inverse implication, that is, whether any $g b$-ranking is an extension of a dichotomous preference $R$ on $X$ and satisfies IND, LPR and LNR, the answer is yes except with respect to IND. We state this idea formally by means of the following theorem.

\footnotetext{
${ }^{6}$ The proof can also be obtained by substituting IND by the aforesaid weaker condition of separability from Barberà et al. (1991), but since IND is a core necessary condition in all the later results we have opted to maintain it so as to preserve the unity of the model.
} 
Theorem 2 Every gb-ranking satisfies $L P R$ and $L N R$, and is an extension of some dichotomous preference $R$ on $X$.

The proof of Theorem 2 is almost immediately apparent. On the one hand, a $g b$-ranking satisfies LPR and LNR because by its mere definition there are $x, y \in X$ such that $\{x\} \succ \emptyset \succ\{y\}$. On the other hand, in order to prove that there is a dichotomous preference $R$ of which $\succcurlyeq$ is an extension, it suffices to define $R$ by $x R y$ iff $\{x\} \succcurlyeq\{y\} .{ }^{7}$

As a matter of fact, each ranking $\succcurlyeq$ enables two subsets of $X, X^{g}$ and $X^{b}$, to be induced such that $X^{g}=\{x:\{x\} \succ \emptyset\}$ and $X^{b}=\{x: \emptyset \succ\{x\}\}$. The interpretation of $X^{g}$ and $X^{b}$ is straightforward as the sets of good and bad objects, respectively. If $\succcurlyeq$ is also a $g b$-ranking, then $X^{g}$ and $X^{b}$ constitute a partition of $X$ and there is indifference between any two singletons containing good objects and any two singletons containing bad objects. Throughout the sections below we use the notation $X^{g}$ and $X^{b}$ to refer to the subsets of good and bad elements induced as above. Analogously, for any $A \in \mathcal{X}$, we set $A^{g}=A \cap X^{g}$ and $A^{b}=A \cap X^{b}$ so that $\left(A^{g}, A^{b}\right)$ is the partition of $A$ into good and bad elements.

The combination of IND, LPR and LNR also makes it possible to characterize a family of rankings that only computes two numbers in order to compare any pair of sets, namely the number of good elements that they contain (evaluated positively) and the number of bad elements (evaluated negatively). ${ }^{8}$

\footnotetext{
${ }^{7}$ We could have merged Theorems 1 and 2 into the following unique and more compact "if-and-only-if" result, but at the cost of some logical redundancies: Let $\succcurlyeq$ satisfy IND, LPR, and LNR. Then, $\succcurlyeq$ is an extension of a dichotomous preference $R$ over $X$ if and only if it is a $g b$-ranking.

${ }^{8}$ Like Theorem 3, all the theorems and propositions in the next section are stated for any ranking $\succcurlyeq$ that is a $g b$-ranking. By virtue of Theorem 1 , each of these theorems could be restated to hold for any ranking $\succcurlyeq$ "that is an extension of a dichotomous preference
} 
Theorem 3 Let $\succcurlyeq$ be a gb-ranking satisfying IND. Then, for all $A, B \in \mathcal{X}$, (1) $\left(\left|A^{g}\right|>\left|B^{g}\right|\right.$ and $\left.\left|A^{b}\right|<\left|B^{b}\right|\right)$ implies $A \succ B$,

(2) $\left(\left|A^{g}\right| \geq\left|B^{g}\right|\right.$ and $\left.\left|A^{b}\right| \leq\left|B^{b}\right|\right)$ implies $A \succcurlyeq B$.

Considering the definition of "good" and "bad" objects as before, it is noteworthy that the family characterized in Theorem 3 is a subfamily of the set of separable preference relations over sets. That is, preferences over sets where, given an arbitrary set of objects, the addition of a good object to the set always results in a higher ranked set while the addition of a bad object results in a lower ranked set. ${ }^{9}$

The section concludes with three examples that illustrate the independence of axioms IND, LPR and LNR. The corresponding ranking in each of these examples satisfies all the axioms discussed but one.

$\neg$ (IND): Let $X=\{x, y\}$ and let $\succcurlyeq_{1}$ be defined as follows: $\{x\} \sim_{1}\{x, y\} \succ_{1}$ $\emptyset \succ_{1}\{y\}$. Clearly, $\succcurlyeq_{1}$ does not satisfy IND since $\emptyset \succ_{1}\{y\}$ and $\{x\} \sim_{1}\{x, y\}$. In order to see that the other two axioms are satisfied it suffices in their corresponding formulations to set $A=\emptyset$ and take as added elements $x$ (for LPR) and $y$ (for LNR).

$\neg(\mathrm{LPR})$ : Let $X=\{x, y\}$ and take $\succcurlyeq_{2}$ to be as follows: $\{x\} \sim_{2} \emptyset \succ_{2}\{x, y\} \sim_{2}$ $\{y\}$. This ranking satisfies IND since we have, on the one hand, that $\{x\} \sim_{2} \emptyset$ and $\{x, y\} \sim_{2}\{y\}$, and on the other hand that $\emptyset \succ_{2}\{y\}$ and $\{x\} \succ_{2}\{x, y\}$. Clearly, LNR is fulfilled (take $A=\emptyset$ and $y$ as added element) while LPR is violated since $\{x, y\} \sim_{2}\{y\}\left(A=\{y\}\right.$ is ruled out), $\{x\} \sim_{2}\{x, y\}(A=\{x\}$ is ruled out), and $\{x\} \sim_{2} \emptyset, \emptyset \succ_{2}\{y\}$ ( $A=\emptyset$ is ruled out).

$\neg$ (LNR): Let $X=\{x, y\}$ and consider the ranking $\succcurlyeq_{3}$ defined as follows:

$R$ on $X$ and satisfies IND, LPR and LNR". We have chosen the former formulation for the sake of fluency.

${ }^{9}$ Separable preferences are commonly used as a primitive in the analysis of voting situations (cf. Barberà et al. (1991), Berga et al. (2004), Ju (2003, 2005)) and coalition formation games (cf. Burani and Zwicker (2003), Dimitrov et al. (2006)). 
$\{x\} \sim_{3}\{x, y\} \succ_{3} \emptyset \sim_{3}\{y\}$. Following arguments analogous to those for $\succcurlyeq_{2}$, it is easy to check that $\succcurlyeq_{3}$ satisfies IND and LPR but not LNR.

Notice that $\succcurlyeq_{1}$ is a $g b$-ranking, while $\succcurlyeq_{2}$ and $\succcurlyeq_{3}$ are not, the reason being that $\{x\} \sim_{2} \emptyset$ and $\emptyset \sim_{3}\{y\}$.

\section{Characterization results}

On the basis of the core axioms presented in Section 3, we now provide axiomatic characterizations of several rules that belong to the above described family.

\subsection{Pairwise offsetting}

The next axiom imposes the existence of a pair of elements in $X$ that, when added to a set, make it remain indifferent.

Local Indifference (LI): There exists $A \in \mathcal{X}$, and $x, y \in X \backslash A$ such that $A \cup\{x, y\} \sim A$.

Like LPR and LNR, LI is an axiom of a "local" nature in the sense that for it to hold it suffices to find a set $A$ and a pair of elements, $x$ and $y$, such that $A \cup\{x, y\} \sim A$. Next we present two propositions that help to suitably interpret LI.

Proposition 4 Let $\succcurlyeq$ be a gb-ranking satisfying IND. If $A \cup\{x, y\} \sim A$ holds for some $A \in \mathcal{X}$ and $x, y \in X \backslash A$, then either $\left[x \in X^{g}\right.$ and $\left.y \in X^{b}\right]$ or $\left[y \in X^{g}\right.$ and $\left.x \in X^{b}\right]$.

What Proposition 4 says is that if IND holds then the corresponding pair of elements added to $A$ should be such that one element is good and the other one is bad. Proposition 4 then helps to interpret LI as an axiom that, 
when combined with IND, imposes at a certain point in the entire ranking over sets that a good element and a bad one exactly offset each other. As shown below, the pair of elements proposed may consist of any good element and any bad element, provided that LI is combined with IND.

Proposition 5 Let $\succcurlyeq$ be a gb-ranking satisfying IND and LI. Then $A \sim$ $A \cup\{x, y\}$ holds for $A \in \mathcal{X}, x \in X^{g} \backslash A$, and $y \in X^{b} \backslash A$.

This kind of offsetting between good and bad elements mentioned above makes sense in many contexts. Imagine, for example, that the problem faced is to form an assembly, committee or jury of arbitrary size where decisions are to be taken by majority vote, and "goodness" or "badness" of members depends on whether they intend to cast their vote against or in favor of the decision maker's interests (belonging to a certain party or country, for example, could be information liable to build up such expectations in some political assemblies). Similarly, in group formation problems a member of a group (for example a club) could be willing to accept what he/she considers a bad new candidate provided that a new good candidate is also accepted. This could also include the problem of allocating working teams in an organization.

Finally, if sets are interpreted as sets of uncertain (dichotomous) possible outcomes in a context of complete uncertainty or ignorance (where no information about the probabilities or likelihood of the outcomes is available), it might prove reasonable for the addition of a potentially bad outcome to be offset by the addition of a potentially good one.

The next theorem shows that IND and LI also serve to characterize the specific $g$ b-ranking $\succcurlyeq_{d}$ (difference rule) defined as follows: ${ }^{10}$ For all $A, B \in \mathcal{X}$,

$$
A \succcurlyeq_{d} B \text { iff }\left|A^{g}\right|-\left|A^{b}\right| \geq\left|B^{g}\right|-\left|B^{b}\right| .
$$

Theorem 6 A gb-ranking satisfies IND and LI if and only if it is $\succcurlyeq_{d}$.

\footnotetext{
${ }^{10}$ See also Dimitrov et al. (2004).
} 
According to Theorem 6 any $g b$-ranking that satisfies IND and LI, (or, alternatively, any extension of a dichotomous preference $R$ over $X$ that satisfies IND, LPR, LNR and LI), necessarily evaluates sets by means of the difference between the number of good and bad elements.

The following examples show that IND and LI are independent axioms even within the domain of $g b$-rankings:

$\neg$ (IND): Let $X=\{x, y, z\}$ and take the $g b$-ranking $\succcurlyeq_{4}$ defined as follows: $\{x, y\} \sim_{4}\{x\} \sim_{4}\{y\} \succ_{4} \emptyset \succ_{4}\{x, y, z\} \sim_{4}\{z\} \sim_{4}\{y, z\} \succ_{4}\{x, z\}$. This ranking does not satisfy IND as $\{x\} \sim_{4}\{y\}$ and $\{y, z\} \succ_{4}\{x, z\}$. However, LI is satisfied since in its formulation we can take $A=\{z\}$ and check that $\{x, y, z\} \sim_{4}\{z\}$ holds.

$\neg(\mathrm{LI})$ : Let $\succcurlyeq_{5}$ be the $g b$-ranking defined as follows:

$$
A \succcurlyeq_{5} B \text { iff }\left\{\begin{array}{l}
\left|A^{b}\right|<\left|B^{b}\right| \\
\text { or } \\
\left|A^{b}\right|=\left|B^{b}\right| \text { and }\left|A^{g}\right| \geq\left|B^{g}\right| .
\end{array}\right.
$$

The check for $\succcurlyeq_{5}$ satisfying IND is left to the reader. As for LI, take $X=$ $\{x, y\}$ with $X^{g}=\{x\}$ and $X^{b}=\{y\}$. Then $\{x\} \succ_{5} \emptyset \succ_{5}\{x, y\} \succ_{5}\{y\}$ in clear violation of LI since no two sets are indifferent.

\subsection{Generalized offsetting}

Consider the following weaker version of LI.

Weak Local Indifference (WLI): There exist $A \in \mathcal{X}$ and $C \in \mathcal{X} \backslash \emptyset$ such that $A \cap C=\emptyset$ and $A \cup C \sim A$.

The axiom simply says that, for some set $A$, there exists another separate set $C$ such that $A \cup C$ remains indifferent to $A$. Clearly this is a weaker version of LI because it does not require $C$ to consist of two elements. It is not difficult to obtain results analogous to Propositions 4 and 5 by substituting 
LI by WLI. That is, the set $C$ should contain both good and bad elements, and it can be replaced by any set $D$ containing the same number of good and bad elements. As noted in the case of LI, such results make it possible to think of contexts where the decision maker is willing to accept the addition of a certain number $j$ of bad elements only if another number of $k$ good elements is added too. Again, certain group formation problems,or working team allocation problems could serve as examples.

It also turns out that substituting LI by WLI in Theorem 6 results in a generalization of the difference rule that considers the weighted difference between the number of good and bad elements according to a certain set of admissible weights. ${ }^{11}$

Theorem 7 A complete gb-ranking $\succcurlyeq$ satisfies IND and WLI if and only if there exist $\alpha \in\left\{1,2, \ldots,\left|X^{b}\right|\right\}$ and $\beta \in\left\{1,2, \ldots,\left|X^{g}\right|\right\}$ such that, for all $A, B \in \mathcal{X}, A \succcurlyeq B \Leftrightarrow \alpha\left|A^{g}\right|-\beta\left|A^{b}\right| \geq \alpha\left|B^{g}\right|-\beta\left|B^{b}\right|$.

As for the independence of axioms IND and WLI in the domain of complete $g b$-rankings, readers are referred to the examples proposed for Theorem 6. Obviously the example that shows the independence of IND is suitable because WLI is weaker than LI. As for the independence of WLI, the same lexicographic rule used to show the independence of LI is also suitable.

\subsection{Infection}

Imagine a situation where bad elements have the power to infect the good ones. That is, though there could initially be good and bad elements in a set the bad feature eventually spreads to all the elements in the set. This could be the case with disease infections or the transmission of computer viruses for

\footnotetext{
${ }^{11}$ This class of rules based on a weighted difference between good and bad elements is a subclass of another weighted additive rule characterized by Alcantud and Arlegi (2012).
} 
example. Another possibility in the context of evolutionary games would be processes where the spread of a negative behavior, such as corrupt practices, in an "evolutionarily stable strategy" (see Bowles (2004)), thus invading, for example, a community of honest traders or civil servants. In such situations the presence of good elements is desirable but it is reasonable to assume that, given the asymmetry of the infection process (bad elements can infect good ones but not vice-versa), the elimination of bad elements is the top priority.

Without loss of generality, and for the sake of fluency, we stick to another economically meaningful problem that fits the "infection" dynamics described above. Consider a simplified repeated public good game where there are only two possible strategies, i.e. contributing to the public good or not contributing at all. Good objects are therefore those subjects that follow the cooperative strategy, while bad objects are the defectors. Experimental evidence shows that, in general, average contributions decay as rounds progress in repeated public good games. This is in general true across different parameters of the game, as amply demonstrated in the last few decades of experimental study since the papers by Isaac, Walker and Thomas (1984) and by Kim and Walker (1984). ${ }^{12}$ It is generally accepted that the coexistence of selfish subjects with selfish-biased conditional cooperators is what motivates this decay (see Neugebauer et al. (2009), Fischbacher et al. (2001), Fischbacher and Gächter (2010), Ashley et al. (2010) or de Oliveira et al. (2013)). Thus, given the particular structure of the public good game, such a selfish-biased conditional cooperation would lead to a progressive contagion of the defective strategy.

In this context, it is not difficult to imagine situations where one might

\footnotetext{
${ }^{12}$ As a matter of fact, Suleiman and Rapoport (1992) find that the defective strategy spreads more easily in repeated public good games where strategies are dichotomous (full contribution or no contribution at all), which are precisely those that best fit our model, than in the case of more general strategies.
} 
be interested in evaluating, comparing or ranking possible sets of objects (subjects in our example). For instance, a supervisor may be interested in designing working teams, or in a coalitional game, an entering player may be interested in evaluating possible coalitions to enter (for example in a joint venture).

All the axioms in Section 3 (IND, LPR and LNR) make sense under this new class of problems: LPR (LNR) would just say that there exist some group where the inclusion of a cooperative (defector) individual is desirable (undesirable). And IND would say that if a set of players, $A$, is considered to be better than another set of players, $B$, then adding or dropping a cooperative (defector) individual in both sets should not affect the original ranking. But the particularity of the problem enables a new axiom to be presented and discussed that is also reasonable.

Elimination of Bad Elements (EL): Let $A, B \in \mathcal{X}$ and $C \subseteq A$ be such that $C \neq \emptyset$ with $\emptyset \succ\{c\}$ for each $c \in C$. Then for $D \in \mathcal{X}, A \succcurlyeq B \Rightarrow A \backslash C \succ$ $B \cup D$.

Roughly speaking, EL states that if a set $A$ is declared to be better than another set $B$, then the positive effect of removing bad elements from the better set prevails over the addition of any set of elements to the worse set. Thus, the new reduced set (with less bad alternatives) is strictly better than the new enlarged set.

In other words, what EL expresses is that adding new elements to the worse set $B$ (a set of new players, either cooperators or defectors, or new people to a group, either healthy or infected) can never offset the fact that bad elements (defectors or infected subjects, for example), which have the power to contaminate any good element are removed from the set that was already better before their elimination.

As shown below, the replacement of LI by EL in Theorem 6 results in the characterization of the bad-elements-priority rule $\succcurlyeq_{b p}$ which is another 
specific $g b$-ranking. More precisely, for all $A, B \in \mathcal{X}$,

$$
A \succcurlyeq_{b p} B \text { iff }\left\{\begin{array}{l}
\left|A^{b}\right|<\left|B^{b}\right| \\
\text { or } \\
\left|A^{b}\right|=\left|B^{b}\right| \text { and }\left|A^{g}\right| \geq\left|B^{g}\right| .
\end{array}\right.
$$

Clearly, $\succcurlyeq_{b p}$ is a lexicographic $g b$-ranking as it first pays attention to the number of "bad" elements in each of the sets under comparison and, in case of a tie, checks for the corresponding numbers of "good" elements.

Theorem 8 A gb-ranking satisfies IND and EL if and only if it is $\succcurlyeq_{b p}$.

In order to demonstrate the independence of IND and EL, consider the following two examples:

$\neg$ (IND): Take $X=\{x, y, z\}$ and again consider the $g b$-ranking $\succcurlyeq_{4}$ defined above as follows: $\{x, y\} \sim_{4}\{x\} \sim_{4}\{y\} \succ_{4} \emptyset \succ_{4}\{x, y, z\} \sim_{4}\{z\} \sim_{4}$ $\{y, z\} \succ_{4}\{x, z\}$. As already known, $\succcurlyeq_{4}$ does not satisfy IND. In order to check that EL is fulfilled, notice that all sets containing the only "bad" element $z$ (and thus, the only possible candidates to play the role of $A$ in the formulation of EL) are strictly worse than the empty set. Moreover, by removing $z$ from any of them sets are obtained that are all strictly better than the empty set, while adding any additional elements to them always results in sets still containing $z$. Thus, it can be concluded that EL is satisfied. $\neg(\mathrm{EL})$ : Let $\succcurlyeq_{6}$ be the $g b$-ranking defined as follows:

$$
A \succcurlyeq_{6} B \text { iff }\left\{\begin{array}{l}
\left|A^{g}\right|>\left|B^{g}\right|, \\
\text { or } \\
\left|A^{g}\right|=\left|B^{g}\right| \text { and }\left|A^{b}\right| \leq\left|B^{b}\right| .
\end{array}\right.
$$

As in the case of $\succcurlyeq_{5}$, checking that $\succcurlyeq_{6}$ satisfies IND is easy and is left to the reader. In order to see that EL is violated, take $X=\{x, y\}$ with $X^{g}=\{x\}$ and $X^{b}=\{y\}$. It then holds that $\{x\} \succ_{6}\{x, y\} \succ_{6} \emptyset \succ_{6}\{y\}$. By EL, $\{x, y\} \succ_{6} \emptyset$ should imply $\{x\} \succ_{6}\{x\}$, a contradiction. 


\subsection{Opportunity sets}

Sets can also be interpreted as opportunity sets. Consider a situation where the decision maker has the power to select one of the elements in the set. In other words, alternatives are mutually exclusive and it is the decision maker who determines the final outcome. As a consequence, if there are both good and bad elements in the set, the decision maker always has the possibility of not choosing the bad ones.

Moreover, assume that the decision maker has a preference for freedom of choice. This means that, independently of which alternative is finally chosen, the availability of others has, for some reason, some value for the decision maker. In particular, Sen (1988) distinguishes two kinds of reason why freedom of choice is valuable. On the one hand, freedom of choice may have an instrumental, or consequentialist, value, meaning that having more opportunities from which to choose is a way of achieving something (for example, greater indirect utility, or the development of human faculties by the exercise of choosing). On the other hand, as discussed by Sen (1988, 1991) and Sugden (1998), freedom of choice may also have an intrinsic value, according to which the mere availability of alternatives to choose from has a value as an end in its own right, independently of any other goal that they may help to achieve.

The paradigm of a ranking of opportunity sets that is based uniquely on an intrinsic value of freedom of choice is the cardinalist ranking, axiomatically characterized by Pattanaik and Xu (1990), where sets are ordered simply according to their number of alternatives, whatever the quality of those alternatives is. Later papers such as Bossert et al. (1994) characterize rules that are compatible with the assumption of an intrinsic value of freedom of choice but also consider the quality of alternatives and the indirect utility that the set may provide, that is, both the intrinsic and the instrumental value of freedom of choice are considered. 
Next we propose an axiom that makes full sense under both the intrinsic and the instrumental assumptions about the value of freedom of choice.

Enlargement with Good Elements (EN): Let $A, B \in \mathcal{X}$ and $C \subseteq X \backslash A$ be such that $C \neq \emptyset$ with $\{c\} \succ \emptyset$ for each $c \in C$. Then for $D \in \mathcal{X}$, $A \succcurlyeq B \Rightarrow A \cup C \succ B \backslash D$.

In words, and considering the freedom of choice context in which the axiom is proposed, this means that if one set $A$ provides (weakly) more freedom of choice than another set $B$ then the addition of further good elements to $A$ will make it strictly better than any subset of $B$ in terms of freedom.

Clearly, EN is suitable in an environment where freedom of choice has an intrinsic value. From this perspective, any enlargement of a set should involve an increase of the intrinsic freedom that it provides for the simple reason that there are more opportunities to choose from. Therefore, if $A \succcurlyeq B$ any proper superset of $A$ would contain a greater intrinsic freedom of choice than any subset of $B$.

But if, following Bossert et al. (1994), it is believed that quality should also be valuable, EN is still suitable because the enlargement of $A$ takes place exclusively with good options.

A meaningful special situation where freedom of choice has an instrumental value is the so-called preference for flexibility problem (see Kreps (1979) for the first axiomatic foundation of the concept of flexibility, also Arlegi and Nieto (2001a, 2001b), and Ahn and Sarver (2013) among many others). In such a situation the decision is made in two stages. In the first an opportunity set is preselected (or evaluated), and in the second the final alternative is picked from the previously preselected set. Moreover, although the decision maker could have preferences defined over the alternatives at the first step they are not definite, so his/her own final preferences when the final choice has to be made are uncertain for him/her in the first stage. As a consequence, the decision maker would like to maintain more alternatives in the 
first stage decision process as long as this helps to dissipate the uncertainty about the preferences in the second stage. More precisely, the availability of alternatives at the first stage of the decision process is valuable in that they could be maximal for some admissible configuration of the future preferences.

For example, imagine that in the first stage the Health System determines the set of potential candidates for a transplant of a recently received organ based uniquely on the compatibility of their blood types. The final objective is to minimize the probability of rejection, and the final decision depends on tests to be conducted on the shortlisted candidates. In this case, any candidate with the compatible blood type adds flexibility to the set to be preselected. Another example would be that of a vegetarian who has to make a reservation at a restaurant some days in advance based on the menu that the restaurant offers, but who is not sure about his/her particular preferences so many days in advance. In this case (assuming that, in principle, he/she is willing to eat any kind of vegetarian food) the presence of any vegetarian dish in the menu adds flexibility to his/her choice.

The same structure applies to problems where an agent has to select a set of alternatives for another agent, who takes the final decision, and the former is not certain about the latter's preferences. For example, a human resources company that has to shortlist candidates for a firm which will eventually hire one of them after a personal interview.

Our assumption of prior dichotomous information fits well into preference for flexibility problems. It is reasonable to consider contexts where, in the first stage, the decision maker is only able to discriminate between acceptable and non acceptable alternatives (blood-type compatible patients for a transplant, vegetarian dishes or job candidates with a certain qualification).

It is thus clear that adding new "acceptable" alternatives to a set $A$ increases the value of the set in terms of flexibility (there are more chances for the enlarged set to contain a potential maximal alternative). Meanwhile, 
no subset of a set $B$ can provide more flexibility than $B$. Consequently EN becomes compelling: if $A \succcurlyeq B$, and $C$ consists only of acceptable alternatives, $A \cup C \succ B \backslash D$ for any set $D$.

In combination with IND, axiom EN enables a lexicographic $g b$-ranking to be characterized that is dual with respect to $\succcurlyeq_{b p}$. We call it the goodelements-priority rule, denote it by $\succcurlyeq_{g p}$, and define it as follows. For all $A, B \in \mathcal{X}$

$$
A \succcurlyeq_{g p} B \text { iff }\left\{\begin{array}{l}
\left|A^{g}\right|>\left|B^{g}\right|, \\
\text { or } \\
\left|A^{g}\right|=\left|B^{g}\right| \text { and }\left|A^{b}\right| \leq\left|B^{b}\right| .
\end{array}\right.
$$

Theorem 9 A gb-ranking satisfies IND and EN if and only if it is $\succcurlyeq_{g p}$.

The theorem says that in a dichotomous environment the good-elementspriority rule is characterized by combining EN and IND.

An important remark is in order regarding Theorem 9. We have claimed that EN fits well with both the intrinsic and the instrumental value of freedom of choice. However, Theorem 9 incorporates two assumptions besides EN: The first is IND, which in principle is not inconsistent with either view about the value of freedom of choice. The second is that the rule characterized is a $g b$-ranking which, by Theorem 2, implies that it satisfies LNR. This axiom could in fact come into conflict with the assumption of an intrinsic value of freedom of choice because from a purist view even the addition of a bad alternative increases the possible choices of the decision maker. Consequently we tend to believe that the combination of all the assumptions in Theorem 9 is more suitable under the instrumental consideration of freedom.

Moreover, according to $\succcurlyeq_{g p}$, not only are bad alternatives disregarded to evaluate sets but they are also evaluated negatively. Again this is mainly as a consequence of LNR, and suggests that the rule fits in contexts where the consideration of an instrumental value of freedom of choice is accompanied by 
a cost for the presence of bad alternatives in the set. For instance, the cost of conducting the final medical tests on the potential receivers of a transplant, the cost of the final personal interviews for the firm in the job candidates case, or the cost of ignoring the non-vegetarian dishes in the restaurant example.

However, the interpretation of the sets as opportunity sets is not the only one under which the good-elements-priority rule may make sense. Coming back to the example of repeated public good games, processes can be imagined that are opposite to those of infection as described in the previous section. For example certain institutional arrangements such as the implementation of adequate structures of punishment ${ }^{13}$ or the internalization of social norms ${ }^{14}$ may induce the presence of conditional cooperators to a spread of the cooperative strategy, inverse to the infection of defective strategies proposed in the previous section. Also, evolutionary game theory is able to explain the potential for "good" behaviors to spread in a population. For example, Bowles (2004) mentions the expansion in certain tribal communities in Africa of the rejection of ablation.

We finish this section by providing two examples that prove the independence of axioms IND and EN.

$\neg$ (IND): Let $X=\{x, y\}$ and take the $g b$-ranking $\succcurlyeq_{1}$ defined above as follows: $\{x\} \sim_{1}\{x, y\} \succ_{1} \emptyset \succ_{1}\{y\}$. As already shown, $\succcurlyeq_{1}$ violates IND. Axiom EN is satisfied since, given that there is a unique good element $x$, the only sets that can play the role of $A$ in the formulation of $\mathrm{EN}$ are $\emptyset$ and $\{y\}$. By adding $x$ to $\emptyset$ it results that both $\{x\} \succ_{1}\{y\}$ and $\{x\} \succ_{1} \emptyset$. By adding $x$ to $\{y\}$ it results that both $\{x, y\} \succ\{y\}$ and $\{x, y\} \succ \emptyset$. Thus, EN is fulfilled in all the instances where it applies.

$\neg(\mathrm{EN})$ : Take the $g$-ranking $\succcurlyeq_{b p}$. By Theorem 8 we know that it satisfies

\footnotetext{
${ }^{13}$ See, for example, Fehr and Gächter (2000), Gürerk et al. (2006), Rockenbach and Milinski (2006), or Gächter et al. (2008).

${ }^{14}$ See, for example, Masclet et al. (2003) and Bochet et al. (2006).
} 
IND. In order to check that $\mathrm{EN}$ is violated, again take $X=\{x, y\}$ with $X^{g}=\{x\}$ and $X^{b}=\{y\}$. This results in $\{x\} \succ_{b p} \emptyset \succ_{b p}\{x, y\} \succ_{b p}\{y\}$. By EN, $\emptyset \succ_{b p}\{x, y\}$ should imply $\{x\} \succ_{b p}\{x\}$, a contradiction.

\section{Concluding remarks}

This paper aims to contribute to the general body of literature about "ranking sets of objects". In particular, starting from a situation where the agent makes a dichotomous partition of the elements of a given set $X$, we study the problem of extending this information axiomatically to obtain rankings over the power set of $X$. There is a wide variety of interpretations that can be given to this formal problem, and how it is solved depends very much upon the particular interpretation adopted. We propose three axioms (Independence (IND), Local Positive Responsiveness (LPR) and Local Negative Responsiveness (LNR)) that we believe are suitable under all the possible interpretations that we have considered.

First, those three axioms enable two start-up results to be obtained: on the one hand they enable the dichotomous partition to be reinterpreted as made in terms of good and bad elements, and on the other hand they characterize a family of rules for evaluating sets that considers as relevant only the information about the number of good elements and the number of bad elements in those sets, and computes the former positively and the latter negatively.

Secondly, the three axioms constitute the axiomatic core of more particular axiomatic characterizations performed upon more specific interpretations of the problem. When they are combined with Local Indifference (LI) the difference rule is obtained; when they are combined with Weak Local Indifference (WLI) the resulting rule is a weighted difference rule; if LI is replaced by Elimination of Bad Elements (EL) the bad-elements-priority rule is obtained 
and if instead Enlargement with Good Elements (EN) is used the result is a dual rule, the good-elements-priority rule.

The axioms of a "local" nature (LPR, LNR, LI and WLI) that we propose are certainly weak in that they only require the existence of a set and an element (or pair of elements) such that the corresponding property holds. However, together with IND, they enable highly structured characterizations to be obtained. Thus, our results reveal that axiom IND, which at a first sight may seem a rather innocuous technical axiom of separability, and one that is widely used in the literature on ranking sets, in fact imposes much more structure than it initially seems to do. The basic reason is that the axiom rules out any kind of correlation between the elements in a set. One might think about weaker versions of IND that palliate this inconvenience, e.g. using only one-direction implications or the strict preference instead of the weak one. Our main finding in this respect is that such weakenings immediately lead to very unstructured and not very meaningful families of rules, unless other parts of the assumptions in the model are reinforced. Interestingly, it is not difficult to prove that our results are still valid if we use a weaker version of IND $(A \succcurlyeq B \Leftrightarrow A \cup\{x\} \succ B \cup\{x\})$, provided that the focus is on complete binary relations only. Finally, the one-direction implication version $(A \succ B \Rightarrow A \cup\{x\} \succ B \cup\{x\})$ of IND would enable some of the results to be obtained only if combined with the additional assumption that $A \sim(A \backslash\{x\}) \cup\{y\}$ holds for any $A \in \mathcal{X}, x \in A$, and $y \in X \backslash A$ with $\{x\} \sim\{y\}$.

We now briefly describe how our model relates to the work of Alcantud and Arlegi (2012). It is worth mentioning that Alcantud and Arlegi (2012) also assume a certain categorization among the primitive items in terms of "good", "neutral", and "bad", and characterize a weighted additive rule. But there are major differences between the two approaches: To start with, we take a step back in our primitive assumptions in the sense that our starting 
point is just a dichotomous partition of the items à la Brams and Fishburn (1978), with no associated connotations of goodness, neutrality or badness. Secondly, the axioms and results are different. For example, in the current article we introduce, as new characterization results, those of the family of rules in Theorem 3 and the two lexicographic rules. As for the family of weighted additive rules that we characterize in Theorem 7 , it is a subclass of the family characterized by Alcantud and Arlegi (2012) in two senses. On the one hand, Alcantud and Arlegi's general rule includes the lexicographic rules, which are not included in the family characterized in Theorem 7. On the other hand, our additive rule only allows the weights $\alpha$ and $\beta$ to take certain particular values (those expressed in the statement of Theorem 7) while Alcantud and Arlegi's rule allows $\alpha$ and $\beta$ to take any real value. A consequence of this is that in Alcantud and Arlegi's family there are rules that satisfy IND but do not satisfy WLI.

Finally, our model performs an axiomatic analysis based on a partition of the set of objects that, though plausible, is certainly elementary. A natural step forward in this research is to further define the structure of the decision maker's information about the alternatives. For instance, the simple information structure described in this paper could be enriched by embedding a similarity relation (cf. Pattanaik and Xu (2000)) into the sets of good and bad objects. This would enable different subgroups of good (bad) objects to be discriminated and would also enable extensions to the rules characterized here to be considered.

\section{Appendix}

This section sets out the proofs of all the theorems that appear in the text. In what follows, for all $S \in \mathcal{X}$ and all $k \in\{1, \ldots,|S|\},(S)_{k}$ denotes any subset of $S$ whose cardinality is $k$. 
Proof of Theorem 1. We have to prove that if $\succcurlyeq$ is an extension of a dichotomous preference $R$ over $X$ and satisfies LPR, LNR and IND, then $\succcurlyeq$ is a $g b$-ranking. To do this it must be proved that there exists a partition $\left(A_{1}^{*}, A_{2}^{*}\right)$ of $\{X\}, A_{i}^{*} \neq \emptyset, i=1,2$, such that $\{a\} \sim\{b\}$ holds for $\{a\},\{b\} \in$ $A_{i}^{*}, i=1,2$, and $\{a\} \succ \emptyset \succ\{b\}$ holds for $\{a\} \in A_{1}^{*}$ and $\{b\} \in A_{2}^{*}$.

Set $R$ as above and let $\succcurlyeq$ be an extension of it. For $i=1,2$, let $A_{i}^{*}=$ $\left\{\{a\}: a \in A_{i} \neq \emptyset\right\}$ and notice that $\left(A_{1}^{*}, A_{2}^{*}\right)$ is a partition of $\{X\}$ as required. Since $\succcurlyeq$ is an extension of $R,\{a\} \sim\{b\}$ holds for $\{a\},\{b\} \in A_{i}^{*}, i=1,2$, and $\{a\} \succ\{b\}$ holds for $\{a\} \in A_{1}^{*}$ and $\{b\} \in A_{2}^{*}$. Notice further that, by LPR, there are $A \in \mathcal{X}, a \in X \backslash A$ such that $A \cup\{a\} \succ A$, which by applying IND repeatedly implies $\{a\} \succ \emptyset$. Similarly, by LNR, there are $B \in \mathcal{X}, b \in X \backslash B$ such that $B \succ B \cup\{b\}$, which by applying IND repeatedly implies $\emptyset \succ\{b\}$. It can be concluded that $\{a\} \in A_{1}^{*}$ and $\{b\} \in A_{2}^{*}$ should hold. Applying transitivity of $\succcurlyeq$ as many times as necessary, leads to $\left\{a^{\prime}\right\} \succ \emptyset \succ\left\{b^{\prime}\right\}$ which holds for any $\left\{a^{\prime}\right\} \in A_{1}^{*}$ and $\left\{b^{\prime}\right\} \in A_{2}^{*}$.

Before presenting the proof of Theorem 3, we first prove the following four lemmas.

Lemma 10 If $\succcurlyeq$ satisfies IND, then $A \sim(A \backslash\{x\}) \cup\{y\}$ holds for $A \in \mathcal{X}$, $x \in A$, and $y \in X \backslash A$ with $\{x\} \sim\{y\}$.

Proof of Lemma 10. By $\{x\} \sim\{y\}$ the repeated application of IND results in $(A \backslash\{x\}) \cup\{x\} \sim(A \backslash\{x\}) \cup\{y\}$. Thus, $A \sim(A \backslash\{x\}) \cup\{y\}$.

Lemma 11 If $\succcurlyeq$ satisfies IND, then for all $A, B \in \mathcal{X}, x \in X \backslash A$, and $y \in X \backslash B$ with $\{x\} \sim\{y\}$, we have that $A \succcurlyeq B \Leftrightarrow A \cup\{x\} \succcurlyeq B \cup\{y\}$.

Proof of Lemma 11. We start by showing that $A \succcurlyeq B$ implies $A \cup\{x\} \succcurlyeq$ $B \cup\{y\}$. To that end assume first that $x, y \in X \backslash(A \cup B)$. If $A \succcurlyeq B$, then, by IND it holds that $A \cup\{x\} \succcurlyeq B \cup\{x\}$ and $A \cup\{y\} \succcurlyeq B \cup\{y\}$ holds. Given 
that $\{x\} \sim\{y\}$, applying IND repeatedly gives $A \cup\{x\} \sim A \cup\{y\}$. Thus, by transitivity, $A \cup\{x\} \succcurlyeq B \cup\{y\}$.

Assume next that $x \in B$ and $y \in A$. By Lemma 10, $(A \backslash\{y\}) \cup\{x\} \sim A$ and $(B \backslash\{x\}) \cup\{y\} \sim B$. Thus, assuming $A \succcurlyeq B$ and applying transitivity gives $(A \backslash\{y\}) \cup\{x\} \succcurlyeq B$. By IND $x$ can be removed from both sets, which gives us $A \backslash\{y\} \succcurlyeq B \backslash\{x\}$. Now it emerges that $x, y \notin(A \backslash\{y\})$ and $x, y \notin(B \backslash\{x\})$. IND can be applied twice, first with $x$ and secondly with $y$ to obtain $(A \backslash\{y\}) \cup\{x\} \cup\{y\} \succcurlyeq(B \backslash\{x\}) \cup\{x\} \cup\{y\}$, that is, $A \cup\{x\} \succcurlyeq B \cup\{y\}$.

Consider now the case where $x \in B$ and $y \notin A$. If $A \succcurlyeq B$, then by Lemma 10 it results that $(B \backslash\{x\}) \cup\{y\} \sim B$ holds. Therefore, by transitivity, $A \succcurlyeq(B \backslash\{x\}) \cup\{y\}$, and by IND, $A \cup\{x\} \succcurlyeq B \cup\{y\}$. The proof for the last case with $y \in A$ and $x \notin B$ is analogous.

What remains to be shown is the reverse implication of the statement, i.e., that $A \cup\{x\} \succcurlyeq B \cup\{y\}$ implies $A \succcurlyeq B$. Two cases can be distinguised: (i) $y \in A$ and $x \in B$, and (ii) $y \notin A$ or $x \notin B$.

(i) Assume that $y \in A$ and $x \in B$. Then, by IND, $A \cup\{x\} \succcurlyeq B \cup\{y\}$ implies that $(A \backslash y) \cup\{x\} \succcurlyeq B$. Again by IND, the latter implies $A \backslash\{y\} \succcurlyeq B \backslash$ $\{x\}$, and applying IND one more time gives $(A \backslash\{y\}) \cup\{y\} \succcurlyeq(B \backslash\{x\}) \cup\{y\})$. Since, by Lemma $10, B \sim(B \backslash\{x\}) \cup\{y\})$, it results by transitivity that $A \succcurlyeq B$ holds.

(ii) Assume first that $y \notin A$. Since $\{x\} \sim\{y\}$, it follows by the repeated use of IND that $A \cup\{x\} \sim A \cup\{y\}$. Then, by transitivity, $A \cup\{x\} \succcurlyeq B \cup\{y\}$ implies $A \cup\{y\} \succcurlyeq B \cup\{y\}$ and thus, $A \succcurlyeq B$ follows by IND. The proof for the case where $x \notin B$ is analogous.

Lemma 12 If a gb-ranking $\succcurlyeq$ satisfies IND, then $B \cup E \succ B$ holds for $B \in \mathcal{X}$ and $E \subseteq X^{g} \backslash B, E \neq \emptyset$.

Proof of Lemma 12. Take $\succcurlyeq, B$, and $E$ as above and let $E=\left\{e_{1}, \ldots, e_{n}\right\}$. 
Given that $e_{1} \in X^{g},\left\{e_{1}\right\} \succ \emptyset$. Thus, $B \cup\left\{e_{1}\right\} \succ B$ holds by the repeated use of IND. Applying the same argument $(n-1)$ more times gives $B \cup E \succ B$.

Lemma 13 If a gb-ranking $\succcurlyeq$ satisfies IND, then $B \cup E \prec B$ holds for $B \in \mathcal{X}$ and $E \subseteq X^{b} \backslash B, E \neq \emptyset$.

Proof of Lemma 13. The proof is analogous to the proof of Lemma 12.

Proof of Theorem 3. (1) Assume that $\left|A^{g}\right|>\left|B^{g}\right|$ and $\left|A^{b}\right|<\left|B^{b}\right|$. If $\left|B^{g}\right|=0$ (i.e., $B^{g}=\emptyset$ ), $A^{g} \succ \emptyset$ follows from Lemma 12 with $A^{g}$ in the role of $E$, i.e., the result is $A^{g} \succ B^{g}$. If $\left|B^{g}\right|=s>0$, starting from $\emptyset \sim \emptyset$, the application of Lemma $11 s$-times results in $\left(A^{g}\right)_{s} \sim B^{g}$. Lemma 12, with $A^{g} \backslash\left(A^{g}\right)_{s}$ in the role of $E$, gives $A^{g} \succ\left(A^{g}\right)_{s}$. This, by transitivity, results in $A^{g} \succ B^{g}$. Therefore, whether $B^{g} \neq \emptyset$ or $B^{g}=\emptyset$, the result is that $A^{g} \succ B^{g}$.

By Lemma 13 and $\left|B^{b}\right|>0$ (i.e., $B^{b} \neq \emptyset$ ), it holds that $B^{g} \succ B$. If $A^{b}=\emptyset$, then by transitivity $A \succ B$. Assume now that $\left|A^{b}\right|=v>0$. Starting from $A^{g} \succ B^{g}$ and applying Lemma $11 v$-times, $A \succ B^{g} \cup\left(B^{b}\right)_{v}$ is obtained. By Lemma 13, with $B^{b} \backslash\left(B^{b}\right)_{v}$ in the role of $E, B^{g} \cup\left(B^{b}\right)_{v} \succ B$, and by transitivity, $A \succ B$.

(2) The case in which $\left|A^{g}\right|>\left|B^{g}\right|$ and $\left|A^{b}\right|<\left|B^{b}\right|$ is proved in the previous paragraph. Thus, the three remaining possible cases can be distinguished:

(2.1) $\left|A^{g}\right|>\left|B^{g}\right|$ and $\left|A^{b}\right|=\left|B^{b}\right|$,

(2.2) $\left|A^{g}\right|=\left|B^{g}\right|$ and $\left|A^{b}\right|<\left|B^{b}\right|$, and

(2.3) $\left|A^{g}\right|=\left|B^{g}\right|$ and $\left|A^{b}\right|=\left|B^{b}\right|$.

(2.1) As in the first part of the proof, it can be proved by using reflexivity, Lemma 11 and Lemma 12, that $A^{g} \succ B^{g}$. By Lemma 11 repeated $\left|A^{b}\right|=$ $\left|B^{b}\right|$-times, $A \succ B$.

(2.2) Let $\left|A^{b}\right|=u \geq 0$. By reflexivity, $\emptyset \sim \emptyset$, and applying Lemma $11 u$-times, $A^{b} \sim\left(B^{b}\right)_{u}$. By Lemma 13, with $B^{b} \backslash\left(B^{b}\right)_{u}$ in the role of $E$, 
$\left(B^{b}\right)_{u} \succ B^{b}$. By transitivity, $A^{b} \succ B^{b}$. Applying Lemma $11\left|A^{g}\right|=\left|B^{g}\right|-$ times, $A \succ B$.

(2.3) By reflexivity, $\emptyset \sim \emptyset$, and applying Lemma $11\left|A^{g}\right|=\left|B^{g}\right|$-times, $A^{g} \sim B^{g}$. Again by Lemma 11 applied $\left|A^{b}\right|=\left|B^{b}\right|$-times, $A \sim B$.

Proof of Proposition 4. Notice that it is not possible for both $x$ and $y$ to belong to $X^{g}$, because in that case, $A \cup\{x, y\} \succ A$ would follow by Lemma 12. Similarly, by Lemma 13 , it is impossible for both $x$ and $y$ to belong to $X^{b}$.

Proof of Proposition 5. Take $A, x \in X^{g} \backslash A$, and $y \in X^{b} \backslash A$, as in the statement of the proposition. By LI it is known that there is a set $B \in \mathcal{X}$ and a pair of elements $c, d \in X \backslash B$ such that $B \cup\{c, d\} \sim B$. By Proposition 4 either $\left[c \in X^{g}\right.$ and $\left.d \in X^{b}\right]$ or $\left[d \in X^{g}\right.$ and $c \in X^{b}$. W.l.o.g., let $c \in X^{g}$ and $d \in X^{b}$. By IND repeatedly applied, $\{c, d\} \sim \emptyset$. Since $c, x \in X^{g}$ and $d, y \in X^{b}$, it results by the definition of a $g$ b-ranking that both $\{c\} \sim\{x\}$ and $\{d\} \sim\{y\}$ hold. By Lemma 11, $\{c, d\} \sim\{x, y\}$ and by transitivity, $\{x, y\} \sim \emptyset$. Finally, by IND repeatedly applied, $A \sim A \cup\{x, y\}$ is reached.

In order to prove Theorem 6, consider first the following lemma.

Lemma 14 If a gb-ranking $\succcurlyeq$ satisfies IND and LI, then $A \sim B$ holds for $A, B \in \mathcal{X}$ such that $A \cap E=\emptyset$ and $B=A \cup E$ for some $E \in \mathcal{X}$ with $\left|E^{g}\right|=\left|E^{b}\right|$.

Proof of Lemma 14. By the repeated application of Proposition 5.

Proof of Theorem 6. It can be easily checked that $\succcurlyeq_{d}$ satisfies IND and LI. Assume now that a $g$ b-ranking $\succcurlyeq$ satisfies IND and LI. It has to be proved that, for all $A, B \in \mathcal{X}$,

(1) $\left|A^{g}\right|-\left|A^{b}\right|>\left|B^{g}\right|-\left|B^{b}\right|$ implies $A \succ B$, and

(2) $\left|A^{g}\right|-\left|A^{g}\right|=\left|B^{g}\right|-\left|B^{b}\right|$ implies $A \sim B$. 
Let $\left|A^{g}\right|=r,\left|B^{g}\right|=s,\left|A^{b}\right|=u,\left|B^{b}\right|=v$.

(1) In this case $r-u>s-v$. Consider the following three possible cases:

(1.1) $r>u$ and $s>v$,

(1.2) $r>u$ and $s \leq v$,

(1.3) $r \leq u$ and $s<v$.

(1.1) Assume that $r>u$ and $s>v$. By reflexivity, $\emptyset \sim \emptyset$. By Lemma 14, $\left(A^{g}\right)_{u} \cup A^{b} \sim \emptyset$. Also by Lemma $14,\left(B^{g}\right)_{v} \cup B^{b} \sim \emptyset$. Thus, by transitivity, $\left(A^{g}\right)_{u} \cup A^{b} \sim\left(B^{g}\right)_{v} \cup B^{b}$. Given that $r-u>s-v$, by Lemma 11 applied $(s-v)$-times $\left(A^{g}\right)_{u+s-v} \cup A^{b} \sim\left(B^{g}\right)_{v+s-v} \cup B^{b}$, i.e., $\left(A^{g}\right)_{u+s-v} \cup A^{b} \sim B$. By Lemma 12, $A \succ\left(A^{g}\right)_{u+s-v} \cup A^{b}$, and by transitivity, $A \succ B$.

(1.2) Let $r>u$ and $s \leq v$. Proceeding as in case (1.1), by reflexivity, Lemma 14 and transitivity give $\left(A^{g}\right)_{u} \cup A^{b} \sim B^{g} \cup\left(B^{b}\right)_{s}$. By Lemma 12, $A \succ\left(A^{g}\right)_{u} \cup A^{b}$, and, if $s<v$, by Lemma 13,

$$
B^{g} \cup\left(B^{b}\right)_{s} \succ B
$$

If $s=v, B^{g} \cup\left(B^{b}\right)_{s}=B$. In any case, by transitivity, $A \succ B$.

(1.3) Let $r \leq u$ and $s<v$. As before, by reflexivity, Lemma 14 and transitivity result in $A^{g} \cup\left(A^{b}\right)_{r} \sim B^{g} \cup\left(B^{b}\right)_{s}$. Since $r-u>s-v$, then $u-r<v-s$. Then Lemma 11 can be applied $(u-r)$-times to give $A^{g} \cup$ $\left(A^{b}\right)_{r+u-r} \sim B^{g} \cup\left(B^{b}\right)_{s+u-r}$. That is, $A \sim B^{g} \cup\left(B^{b}\right)_{s+u-r}$. By Lemma 13,

$$
B^{g} \cup\left(B^{b}\right)_{s+u-r} \succ B .
$$

Then, by transitivity, $A \succ B$.

(2) In this case $r-u=s-v$. If $r \geq u(s \geq v)$, then, as in case (1), by reflexivity, Lemma 14 and transitivity the following is obtained:

$$
\left(A^{g}\right)_{u} \cup A^{b} \sim\left(B^{g}\right)_{v} \cup B^{b}
$$

and by Lemma 11 applied $(r-u)(=s-v)$-times, $A \sim B$. 
If $r<u(s<v)$, then, by reflexivity, Lemma 14 and transitivity the following is obtanied

$$
A^{g} \cup\left(A^{b}\right)_{r} \sim B^{g} \cup\left(B^{b}\right)_{s},
$$

and by Lemma 11 applied $(u-r)(=v-s)$-times, $A \sim B$.

Proof of Theorem 7. We first show that any complete $g b$-ranking satisfying IND and WLI satisfies the two axioms given below, and then utilize a result in Alcantud and Arlegi (2012) in order to complete our proof. The two new axioms are the following:

Symmetry within categories (SWC) For $a, b \in X$, if $(\{a\} \succ \varnothing$ and $\{b\} \succ \varnothing)$ or $(\varnothing \succ\{a\}$ and $\varnothing \succ\{b\})$, then $\{a\} \sim\{b\}$.

Independence (IN) For $A, B, C, D \in \mathcal{X}$ such that $(A \cup B) \cap(C \cup D)=\varnothing$ and $C \sim D, A \succcurlyeq B$ if and only if $A \cup C \succcurlyeq B \cup D$.

Notice that any $g b$-ranking satisfies SWC by its very definition. As shown in the next lemma, axioms IND and IN are equivalent.

Lemma 15 A ranking satisfies IND if and only if it satisfies IN.

Proof of Lemma 15. Assume first that $\succcurlyeq$ satisfies IND. It has to be proved that it also satisfies IN, that is, for $A, B, C, D \in \mathcal{X}$ such that $(A \cup B) \cap(C \cup$ $D)=\varnothing$ and $C \sim D, A \succcurlyeq B$ if and only if $A \cup C \succcurlyeq B \cup D$. Assume first that $A \succcurlyeq B$. By IND repeatedly applied $A \cup C \succcurlyeq B \cup C$. Moreover, given that $C \sim D$, applying repeatedly IND it can also be obtained that $B \cup C \sim B \cup D$. Then, by transitivity, $A \cup C \succcurlyeq B \cup D$. In order to prove the inverse implication, assume now that $A \cup C \succcurlyeq B \cup D$. We know by IND that $B \cup C \sim B \cup D$. Therefore, by transitivity $A \cup C \succcurlyeq B \cup C$, and again applying IND repeatedly $A \succcurlyeq B$ is obtained.

Assume next that $\succcurlyeq$ satisfies IN. Take $A, B \in \mathcal{X}$ and $x \in X \backslash(A \cup B)$ as in the formulation of IND. By setting $C=D=\{x\}$ and noticing that 
$\{x\} \cap(A \cup B)=\emptyset$, IN can be applied to conclude that $A \succcurlyeq B$ holds if and only if $A \cup\{x\} \succcurlyeq B \cup\{x\}$ as was to be proved.

Hence, for any complete $g b$-ranking satisfying IND the following result (Theorem 4.6 in Alcantud and Arlegi (2012)) applies.

Theorem (Alcantud and Arlegi (2012)) A complete ranking $\succcurlyeq$ satisfies IN and $S W C$ if and only if there exist $\alpha, \beta \in \mathbb{R}_{++}$such that for all $A, B \in \mathcal{X}$, $A \succcurlyeq B \Leftrightarrow \alpha\left|A^{g}\right|-\beta\left|A^{b}\right| \geq \alpha\left|B^{g}\right|-\beta\left|B^{b}\right|$.

We finally show that if, additionally, $\succcurlyeq$ satisfies WLI, $\alpha \in\left\{1,2, \ldots,\left|X^{b}\right|\right\}$ and $\beta \in\left\{1,2, \ldots,\left|X^{g}\right|\right\}$. By WLI it is known that there are $A \in \mathcal{X}$ and $C \in \mathcal{X} \backslash \emptyset$ such that $A \cap C=\emptyset$ and $A \cup C \sim A$. By IND repeatedly applied this implies $C \sim \emptyset$. Since $C \neq \emptyset$, it follows in view of Lemma 12 and Lemma 13 that $C \sim \emptyset$ is only possible if there are $x, y \in C$ such that $\{x\} \succ \emptyset$ and $\emptyset \succ\{y\}$. By Theorem 4.6 in Alcantud and Arlegi (2012), $C \sim \emptyset$ implies $\alpha\left|C^{g}\right|-\beta\left|C^{b}\right|=0$ which holds if $\alpha=\left|C^{b}\right|$ and $\beta=\left|C^{g}\right|$. Obviously, $\left|C^{b}\right| \in\left\{1,2, \ldots,\left|X^{b}\right|\right\}$ and $\left|C^{g}\right| \in\left\{1,2, \ldots,\left|X^{g}\right|\right\}$. This completes the proof of the sufficient part of Theorem 7 .

To prove the necessary part of the theorem it must be checked that if $\succcurlyeq$ is a complete $g b$-ranking and there are $\alpha \in\left\{1,2, \ldots,\left|X^{b}\right|\right\}$ and $\beta \in\left\{1,2, \ldots,\left|X^{g}\right|\right\}$ such that, for all $A, B \in \mathcal{X}, A \succcurlyeq B \Leftrightarrow \alpha\left|A^{g}\right|-\beta\left|A^{b}\right| \geq \alpha\left|B^{g}\right|-\beta\left|A^{b}\right|$, then $\succcurlyeq$ satisfies IND and WLI. The fulfillment of IND follows directly from the additive structure of the rule. As for WLI, note that there is always a set $C$ such that $\left|C^{g}\right|=\beta$ and $\left|C^{b}\right|=\alpha$. This means that $\alpha\left|C^{g}\right|-\beta\left|C^{b}\right|=0$ and thus, $C \sim \emptyset$ holds. Hence, there are $A \in \mathcal{X}(A=\emptyset)$ and $C \in \mathcal{X} \backslash \emptyset$ such that $A \cap C=\emptyset$ and $A \cup C \sim A$, as required for WLI to hold.

Proof of Theorem 8. It is not difficult to check that $\succcurlyeq_{b p}$ satisfies the two axioms. Assume now that a $g b$-ranking $\succcurlyeq$ satisfies IND and EL. It must be proved that, for all $A, B \in \mathcal{X}$,

(1) $\left|A^{b}\right|<\left|B^{b}\right|$ implies $A \succ B$, 
(2) $\left(\left|A^{b}\right|=\left|B^{b}\right|\right.$ and $\left.\left|A^{g}\right|>\left|B^{g}\right|\right)$ implies $A \succ B$, and

(3) $\left(\left|A^{b}\right|=\left|B^{b}\right|\right.$ and $\left.\left|A^{g}\right|=\left|B^{g}\right|\right)$ implies $A \sim B$.

(1) Let $\left|A^{b}\right|=k$ and $\left|B^{b}\right|=k+j$, with $j \geq 1$. By reflexivity $B^{b} \succsim B^{b}$ and by EL $\left(B^{b}\right)_{k} \succ B^{b} \cup B^{g}$, that is, $\left(B^{b}\right)_{k} \succ B$. By Theorem $3 A^{b} \sim\left(B^{b}\right)_{k}$, therefore, by transitivity $A^{b} \succ B$. If $A^{g}=\emptyset$, then $A \succ B$ as desired. If $A^{g} \neq \emptyset$ then $A \succ A^{b}$ by Lemma 12 , and by transitivity $A \succ B$.

(2) Let $\left|A^{g}\right|>\left|B^{g}\right|=s$. By Theorem 3, $A^{b} \cup\left(A^{g}\right)_{s} \sim B$. By Lemma 12, $A \succ A^{b} \cup\left(A^{g}\right)_{s}$, and by transitivity, $A \succ B$.

(3) Again by Theorem 3, $A \sim B$.

Proof of Theorem 9. It is not difficult to check that $\succcurlyeq_{g p}$ satisfies the two axioms. Assume now that a $g b$-ranking $\succcurlyeq$ satisfies IND and EN. It must be proved that, for all $A, B \in \mathcal{X}$,

(1) $\left|A^{g}\right|>\left|B^{g}\right|$ implies $A \succ B$,

(2) $\left(\left|A^{g}\right|=\left|B^{g}\right|\right.$ and $\left.\left|A^{b}\right|<\left|B^{b}\right|\right)$ implies $A \succ B$, and

(3) $\left(\left|A^{g}\right|=\left|B^{g}\right|\right.$ and $\left.\left|A^{b}\right|=\left|B^{b}\right|\right)$ implies $A \sim B$.

(1) Let $\left|B^{g}\right|=k$ and $\left|A^{g}\right|=k+j$. Three possibilities are considered: First, if $\left|A^{b}\right|<\left|B^{b}\right|$, by Theorem 3, $A \succ B$. Second, if $\left|A^{b}\right|=\left|B^{b}\right|$, then by Theorem 3, $A^{b} \cup\left(A^{g}\right)_{k} \sim B$. By Lemma 12, $A \succ A^{b} \cup\left(A^{g}\right)_{k}$, and by transitivity, $A \succ B$.

Thirdly, assume that $\left|A^{b}\right|>\left|B^{b}\right|$. Let $n=\left|A^{b}\right|-\left|B^{b}\right|$. Then it is known that there exists a nonempty set $\left\{a_{1}, \ldots, a_{n}\right\} \subset A^{b} \backslash B^{b}$. By construction and Theorem $3\left(A^{g}\right)_{k} \cup A^{b} \sim B \cup\left\{a_{1}, \ldots, a_{n}\right\}$, and by applying EN $A \succ B$ is obtained.

Notice finally that the implications in cases (2) and (3) follow from Theorem 3 and the transitivity of $\succcurlyeq$. 


\section{References}

[1] Ahn, D.S. and T. Sarver (2013): Preference for flexibility and random choice, Econometrica 81(1), 341-361.

[2] Alcantud, J.C.R. and R. Arlegi (2012): An axiomatic analysis of ranking sets under simple categorization, SERIEs 3(1-2), 227-245.

[3] Allport, G.W. (1954): The nature of prejudice. Reading MA: Addison Wesley.

[4] Arlegi, R. (2003): A Note on Bossert, Pattanaik and Xu's: "Choice under complete uncertainty: axiomatic characterization of some decision rules", Economic Theory 22(1), 219-225.

[5] Arlegi, R. (2007): Sequentially consistent rules of choice under complete uncertainty, Journal of Economic Theory 135(1), 131-143.

[6] Arlegi, R. and J. Nieto (2001a): Incomplete preferences and the preference for flexibility, Mathematical Social Sciences 41(2), 151-165.

[7] Arlegi, R. and J. Nieto (2001b): Ranking opportunity sets: an approach based on the preference for flexibility, Social Choice and Welfare 18(1), 23-36.

[8] Ashley, R., S. Ball and C. Eckel (2010): Motives for giving: a reanalysis of two classic public goods experiments, Southern Economic Journal $77(1), 15-26$.

[9] Barberà, S., C.R. Barret and P.K. Pattanaik (1984): On some axioms for ranking sets of alternatives, Journal of Economic Theory 33, 301-308.

[10] Barberà, S., W. Bossert, and P. Pattanaik (2004): Ranking sets of objects, in: Barberà, S., P. Hammond, and Ch. Seidl (eds.), Handbook of Utility Theory, Vol. 2, Kluwer Academic Publishers, pp. 893-977. 
[11] Barberà, S., M. Maschler, and J. Shalev (2001): Voting for voters: a model of electoral evolution, Games and Economic Behavior 37, 40-78.

[12] Barberà, S. and P.K. Pattanaik (1984): Extending an order on a set to the power set: some remarks on Kannai and Peleg's approach, Journal of Economic Theory 32, 185-191.

[13] Barberà, S., H. Sonnenschein, and L. Zhou (1991): Voting by committees, Econometrica 59, 595-609.

[14] Berga, D., G. Bergantiños, J. Massó, and A. Neme (2004): Stability and voting by committees with exit, Social Choice and Welfare 23, 229-247.

[15] Bochet, O., Page, T. and L. Putterman (2006): Communication and punishment in voluntary contribution experiments, Journal of Economic Behavior and Organization 60(1), 11-26.

[16] Bogomolnaia, A. and H. Moulin (2004): Random matching under dichotomous preferences, Econometrica 72, 257-279.

[17] Bogomolnaia, A., H. Moulin, and R. Stong (2005): Collective choice under dichotomous preferences, Journal of Economic Theory 122, 165184.

[18] Bossert, W., P.K. Pattanaik and Y. Xu (1994): Ranking opportunity sets: an axiomatic approach, Journal of Economic Theory 63, 326-345.

[19] Bossert, W., P.K. Pattanaik and Y. Xu (2000): Choice under complete uncertainty: axiomatic characterization of some decision rules, Economic Theory 16(2), 295-312.

[20] Bowles, S. (2004): Microeconomics: behavior, institutions, and evolution, Russell Sage Foundation, Princeton; Princeton University Press, Oxford. 
[21] Brams, S. and P. Fishburn (1978): Approval voting, American Political Science Review 72, 831-847.

[22] Brams, S. and P. Fishburn (2002): Voting Procedures. In Arrow, K.J., Sen, A.K. and Suzumura, K., Handbook of Social Choice and Welfare, Vol. 1, Elsevier, pp. 174-236.

[23] Burani, N. and W. S. Zwicker (2003): Coalition formation games with separable preferences, Mathematical Social Sciences 45, 27-52.

[24] de Oliveira, A.C.M., R.T.A. Croson and C. Eckel (2013): One bad apple? Heterogeneity and information in public good provision, Experimental Economics, forthcoming.

[25] Dimitrov, D., P. Borm, R. Hendrickx, and S.-C. Sung (2006): Simple priorities and core stability in hedonic games, Social Choice and Welfare 26, 421-433.

[26] Dimitrov, D., P. Borm, and R. Hendrickx (2004): Good and bad objects: the symmetric difference rule, Economics Bulletin 4(11), 1-7.

[27] Dimitrov, D., S.-C. Sung, and Y. Xu (2007): Procedural group identification, Mathematical Social Sciences 54, 137-146.

[28] Fehr, E. and S. Gächter (2000): Cooperation and punishment in public goods experiments, American Economic Review 90(4), 980-994.

[29] Fischbacher, U. and S. Gächter (2010): Social preferences, beliefs, and the dynamics of free riding in public good experiments, American Economic Review 100(1), 541-556.

[30] Fischbacher, U., S. Gächter, and E. Fehr (2001): Are people conditionally cooperative? Evidence from a public goods experiment, Economics Letters 71(3), 397-404. 
[31] Gächter, S., E. Renner, and M. Sefton (2008): The long run benefits of punishment, Science 322, 1510.

[32] Gürerk, Ö., B. Irlenbusch, and B. Rockenbach (2006): The competitive advantage of sanctioning institutions, Science 312, 108-111.

[33] Isaac, R.M., J.M. Walker, and S.H. Thomas (1984): Divergent evidence on free riding: An experimental examination of possible explanations, Public Choice 43, 113-149.

[34] Ju, B.-G. (2005): An efficiency characterization of plurality social choice on simple preference domains, Economic Theory 26, 115-128.

[35] Ju, B.-G. (2003): A characterization of strategy-proof voting rules for separable weak orderings, Social Choice and Welfare 21, 469-499.

[36] Kasher, A. and A. Rubinstein (1997): On the question 'Who is a J?': a social choice approach, Logique et Analyse 160, 385-395.

[37] Kim, O. and J.M. Walker (1984): The free rider problem: experimental evidence, Public Choice 43, 3-24.

[38] Kreps, D.M. (1979): A representation theorem for preference for flexibility, Econometrica 47, 565-577.

[39] Masclet, D., C. Noussair, M. Villeval, and S. Tucker (2003): Monetary and nonmonetary punishment in the voluntary contributions mechanism, American Economic Review 93(1), 366-380.

[40] Neugebauer, T., J. Perote, U. Schmidt, and M. Loos (2009): Selfishbiased conditional cooperation: on the decline of contributions in repeated public goods experiments, Journal of Economic Psychology 30, $52-60$. 
[41] Nitzan, S. and P.K. Pattanaik (1984): Median-based extensions of an ordering over a set to the power set: an axiomatic characterization, Journal of Economic Theory 34, 252-261.

[42] Pattanaik, P. and Y. Xu (2000): On diversity and freedom of choice, Mathematical Social Sciences 40, 123-130.

[43] Pattanaik, P. and Y. Xu (1990): On ranking opportunity sets in terms of freedom of choice, Recherches Economiques de Louvain 56, 383-390.

[44] Rockenbach, B. and M. Milinski (2006): The efficient interaction of indirect reciprocity and costly punishment, Nature 444, 718-723.

[45] Romero-Medina, A. (2001): More on preferences and freedom, Social Choice and Welfare 18, 179-191.

[46] Samet, D. and D. Schmeidler (2003): Between liberalism and democracy, Journal of Economic Theory 110, 213-233.

[47] Sen, A. (1988): Freedom of choice: concept and content, European Economic Review 32, 269-294.

[48] Sen, A. (1991): Welfare, preference and freedom, Journal of Econometrics $50,15-29$.

[49] Sugden, R. (1998): The metric of opportunity, Economics and Philosophy $14,307-337$.

[50] Suleiman R. and A. Rapoport (1992): Provision of step-level public goods with continuous contribution, Journal of Behavioral Decision Making 5, 133-153. 